\title{
Nanomaterials and Nanorobotics in Dentistry: A Review
}

\author{
Yelda Kasimoglu ${ }^{1}$, Derya Tabakcilar ${ }^{2}$, Zeynep A. Guclu ${ }^{3}$, Seiko Yamamoto-Nemoto ${ }^{4}$, \\ Elif B. Tuna ${ }^{5}$, Bugra Ozen ${ }^{6}$, Tamer Tuzuner ${ }^{7}$, Gokhan Ince ${ }^{8}$
}

${ }^{1}$ Istanbul University, Faculty of Dentistry, Department of Pedodontics, Istanbul, Turkey,

${ }^{2}$ Yeditepe University Faculty of Dentistry, Department of Pediatric Dentistry, Istanbul, Turkey

${ }^{3}$ Erciyes University Faculty of Dentistry, Department of Pedodontics, Kayseri, Turkey

${ }^{4}$ Nihon University School of Dentistry at Matsudo, Department of Pediatric Dentistry, Matsudo, Japan

${ }^{5}$ Istanbul University Faculty of Dentistry, Department of Pedodontics, Istanbul, Turkey

${ }^{6}$ Altınbas University Faculty of Dentistry, Department of Pedodontics, Istanbul, Turkey

${ }^{7}$ Karadeniz Technical University Faculty of Dentistry, Department of Pedodontics, Trabzon, Turkey

${ }^{8}$ Istanbul Teknik University, Department of Computer Engineering, Istanbul, Turkey

Correspondencee-mail to: yelda.kasimoglu@istanbul.edu.tr

\begin{abstract}
Nanotechnology is a branch of science focusing on the manipulation of materials measured on the nanoscale $($ size $=1-100 \mathrm{~nm})$. Recent advances in the field of nanodentistry have resulted in the development of alternative treatment plans for common dental problems, bringing about a paradigm shift in dentistry. Nanorobots, also known as "nanites" or "nanomachines," are theoretical microscopic devices that may be used for the diagnosis and treatment of oral health problems. This paper aims to discuss the latest innovations in the field of nanodentistry.
\end{abstract}

Key words: nanotechnology, nanodentistry, nanomaterials, nanorobotics, dentistry

How to cite this article: Kasimoglu Y, Tabakcilar D, Guclu ZA, Yamamoto-Nemoto S, Tuna EB, Ozen B, Tuzuner T, Ince G. Nanomaterials and nanorobotics in Dentistry: A review. J Dent Indones. 2020;27(2):77-84

\section{INTRODUCTION}

Nanotechnology, a field of science that includes research and development of the manipulation and manufacture of materials and devices measured on the nanometer scale, has been described by the author of "Engines of Creation" as a new wave of technology where engineers can build nanocircuits and nanomachines. ${ }^{1}$ The prefix "nano," derived from the Greek word "nannos" meaning "dwarf," represents one billionth of any unit of measure. ${ }^{2}$ Therefore, one "nanometer" equals to 2-3 atoms or one millionth of a meter $\left(10^{-9}\right.$ meters $){ }^{3,4}$

Nobel laureate Richard P. Feynman first used the term "nanotechnology" in his lecture at the Annual Meeting of the American Physical Society in $1959 .^{5}$ He suggested the existence of small machines such as computers and micro or nanorobots, and claimed that his idea was "a development that cannot be avoided." This later went on to open several windows of opportunity in the field of dentistry, making the vision of nanotechnology an important emerging discipline., ${ }^{1,4-6}$ Commercial applications of nanotechnology include the presence of nanoparticles in cosmetics, sunscreen, food packaging, clothing, and disinfectants since the 2000s. The application of nanotechnology in medicine and dentistry (nanomedicine and nanodentistry) includes the development of systems that deliver drugs and other substances to specific types of cells, thus helping treat diseases and prevent health issues.

This review summarizes the basic concepts of nanotechnology, and introduces nanodentistry by exploring the use of this technology in current dental treatments and materials.

Applications of Nanotechnology in Dental Practice Application of the main principles of nanotechnology in dentistry has led to the development of a new concept, "nanodentistry," which may be defined as the improvement and maintenance of oral health through the use of nanomaterials and biotechnology, including tissue engineering and nanorobotics. ${ }^{7}$ Research in nanoden- 
tistry covers a wide range of areas including diagnosis of diseases (nanodiagnosis), prevention of dental caries (nanoprevention), and dental treatments (nanotreatment).

\section{NanoDiagnosis}

Potential applications of nanorobotics devices in medicine include early detection of diseases such as cancer at the cellular and molecular levels, pharmacokinetics, and detection of biomarkers and pathogens. ${ }^{8}{ }^{8}$ For example, early diagnosis of cancer and monitoring of disease progression has been achieved using a novel type of biosensor surface modification for the highly specific detection of short RNAs. ${ }^{10}$

Life-threatening oral diseases such as oral and pharyngeal cancer ${ }^{11}$ have been listed as the most common type of cancer in many countries, affecting thousands of people annually. Globally, oral cancer alone affects 350,000 people per year, over $90 \%$ of which are diagnosed with oral squamous cell carcinoma (https://oralcancerfoundation.org/facts/2016/). Although patient morbidity and the overall 5-year survival rates have improved, oral squamous cell carcinoma remains the one of the most fatal cancers. ${ }^{12,13}$ In general, oral cancer can be identified clinically through the presence of premalignant symptoms, and its incidence and mortality can be reduced effectively through early detection. Saliva may be used as a non-invasive diagnostic tool for analyzing the biochemical indices of proteomic and genomic markers. ${ }^{13}$

Human saliva includes a subset of 100 nanometers of a type of secretory vesicles, known as "exosomes," which represent a particular set of biomarkers. ${ }^{14}$ Exosomes can carry mRNA and microRNA, and these exosomal mRNAs can be translated into proteins in target cells. Released from the salivary gland epithelium, exosomes have a cell-specific membrane and release proteins surrounded by a lipid bilayer into the salivary fluid through exocytosis. Malignancy and other diseases increase exosome secretion, and tumor antigen increases exosomes linked with cancer cells. Due to their small size, the discovery and characterization of salivary exosomes require precise and quantitative detection tools. The structure of individual vesicles and the molecular surface details of human salivary exosomes have recently been investigated as a potential source of non-invasive biomarkers for oral cancer., ${ }^{8,15,16}$ Commercial exosome isolation kits such as ExoQuick$\mathrm{TC}^{\text {TM }}$ have been developed to allow investigation of these salivary exosomes. ${ }^{17}$

Identifying cancer-related cell changes using polymeric nanoparticles ${ }^{18}$ and, subsequently, detecting cancer at an early stage using minimally invasive techniques greatly improves prognosis. ${ }^{19}$ Applications of nanodiagnosis include sensor systems such as nanoelectro mechanical systems (NEMSs), oral fluid nano-sensor test (OFNASET), and optical nanobiosensors.
NEMS: Nanoelectro mechanical systems (NEMs) transform the biochemical signal and cantilever array sensor into sensitive technology suitable for the detection of bacteria, viruses, and DNA at the molecular level. This technology is useful in diagnosing oral cancer and diabetes mellitus, and also in detecting bacteria, viruses, and fungi. ${ }^{20-22}$ For example, implementation of an "artificial pancreas" integrated system may improve diabetes mellitus management.

OFNASET: Oral fluid nanosensor tests (OFNASET) are micro-electromechanical systems consisting of sensor array chips used for the detection of electrochemical sensors of cancer such as salivary protein and RNA biomarkers. The system is based on connecting target RNA molecules or proteins to the surface of the sensor efficiently and utilizes micro-fluid technology, defined as the automation of analytical laboratory procedures onto a single device or "chip." A combination of four salivary mRNA biomarkers (Oz/ten-m homolog-ODZ, IL-1b, IL-8, and salivary transkriptome-SAT) and two salivary proteomic biomarkers (IL-8 and thioredoxin) has been shown to have high sensitivity and specificity for the detection of oral cancer. ${ }^{13,16}$ Additionally, it can also be used for diagnosis of breast, pancreatic, and lung cancers; Type II diabetes; Alzheimer's disease; and Sjögren's syndrome. ${ }^{23}$

Optical nanobiosensors: These use the principles of optics for the transduction of biochemical interactions into suitable output signals. The fiber-optic tool allows non-invasive analysis of intracellular components such as cytochrome $\mathrm{C}$, which is important for cellular energy production and is a widely recognized protein involved in programmed cell death or apoptosis. . $^{16,24}$

\section{NanoPrevention}

Remineralization represents a major advancement in the clinical management of lost enamel surfaces, and the introduction of nanoparticles in fissure sealants, fluorides, and toothpastes can assist in the prevention of dental caries.

\section{Sealants}

Braun et al. demonstrated that, relative to conventional sealant materials, fissure sealants containing nanofillers exerted minimal effect on laser fluorescence values during caries detection. Therefore, nanomaterials may be used to assess caries progress underneath sealants and to initiate appropriate treatment in a timely manner. However, further research on the properties of these materials and the long-term reliability of these treatment strategies is necessary. ${ }^{13}$

\section{NanoSilver Fluorides}

Although silver diamine fluoride application is a conservative, effective, and non-invasive treatment option for the prevention of dental caries, it often 
causes staining of the teeth through deposition of silver particles on the dentin layer and formation of a silver phosphate layer, resulting in aesthetic deterioration. ${ }^{26}$ This has led to the development of new formulations containing silver nanoparticles that produce decreased staining as the particles are smaller and therefore do not undergo oxidation. Smaller silver particles also have the added benefits of increased surface contact with cariogenic cells. One such new formulation, known as nanosilver fluoride (NSF), contains silver nanoparticles, chitosan, and fluoride, and exhibits antimicrobial properties against Lactobacilli and Streptococcus mutans. Laboratory and clinical studies have shown that NSF is safe to use in humans, is economical, and can be used to arrest active dentin caries in children. ${ }^{27-30}$

\section{NanoHydroxyapatite Toothpastes}

Nanohydroxyapatite toothpastes deliver a nanocrystalline form of hydroxyapatite particles at a size 20-50 nm which is conducive for natural repair. Previous studies have reported that nanohydroxyapatite toothpastes inhibited caries development and exhibited higher remineralizing potential compared to those containing amine fluorides, suggesting that these were effective alternatives to fluoride toothpastes. ${ }^{16,24}$

Some commercially available mouthwashes are formulated using bio-adhesive nanoparticles and are loaded with triclosan. Silver nanoparticles create stable and effective silver products that possess antibacterial properties and can reduce the risk of halitosis and gingivitis caused by bacteria in the mouth. ${ }^{31}$

Nanohydroxyapatite toothpastes are highly bioactive and biocompatible with potassium nitrate, which seals exposed dentinal tubules and creates a protective coating that blocks the transmission of external stimuli leading to tooth sensitivity. ${ }^{32}$ Therefore, biomimetic approaches, such as fluoride-free nanohydroxyapatite toothpastes, represent alternative methods for the resolution of ongoing decay and effective desensitization that quickly relieves symptoms. ${ }^{32,33}$ Inclusion of nanohydroxyapatite in daily products such as toothpastes and mouthwashes can allow management of biofilm formation on the enamel surface and, potentially, repair enamel..$^{34-37}$ Additionally, nanohydroxyapatite dentifrices also have the added benefits of low cost and ease of use. ${ }^{38}$

\section{NanoTreatment}

There is significant growth in the field of science exploring incorporation of nanotechnology in dental treatments, resulting in vast improvements in the materials used for oral therapy.

\section{Restorative Treatment}

\section{Nano Light-Curing Glass Ionomers}

The combination of nanotechnology and fluoraluminosilicate technology was found to greatly improve the wear resistance, esthetics, and polishability of glass ionomer materials. Clinical indications of this material include core build-ups; class I, III, and V restorations; sandwich technique; and restorative treatment of primary teeth. ${ }^{29}$

\section{Nanocomposites}

Nanocomposites have filler particle sizes of 0.1-100 $\mathrm{nm}$, which are difficult to wet with resin but are more suitable than large particles for the achievement of good aesthetics, polishability, and abrasion resistance. Compared with traditional fillers, nanofillers have lower polymerization shrinkage and better mechanical properties such as hardness, tensile strength, compressive strength, and resistance to fracture. ${ }^{39}$

Taking these into consideration, a comprehensive resin system consisting of rheologically modified nanofiller particles that fulfill all esthetic and functional requirements was developed. ${ }^{40}$

Ormocer-based resin is an example of a recently developed nanohybrid resin composite. The clinical performance (evaluated using slightly modified USPHS criteria) of such resin composites in Class II cavities was found to be satisfactory after 4 years of evaluation, and the performance did not vary with the type of adhesive material used. ${ }^{41}$ Another clinical study also reported acceptable performance after 3 years in wide molar restorations. ${ }^{42}$

The use of nanohydroxyapatite in the repair of early carious lesions has been studied extensively. Upon exploring the effects of size and $\mathrm{pH}$ of nanohyroxyapatite particles, Huang et al. found that the remineralization potential increased considerably at $\mathrm{pH}$ values less than 7.0, suggesting that these particles could function effectively as repair materials and anti-caries agents. ${ }^{43}$ Cheng et al. ${ }^{44}$ developed nanocomposites containing calcium fluoride nanoparticles or amorphous calcium phosphate and chlorhexidine, and investigated the biofilm formation and lactic acid production of $S$. mutans. Compared with a commercial composite, the new nanocomposites exhibited a 10-20 fold reduction in biofilm acid production and metabolic activity. ${ }^{41,44,45}$ However, further studies are still necessary in order to optimize nanocomposites and understand their anticaries ability.

Following application of a self-etching adhesive, Deshmuch and Nandlal compared the shear bond strength of traditional composites with those of nanocomposites in healthy and carious dentin, and found that the latter offered higher bond strength. ${ }^{40}$ Premise (KerrHawe Bioggio, Switzerland), Ceram X (Dentsply DeTrey, Konstanz, Germany), Filtek Supreme universal restorative pure nano (3M Espe, St. Paul, USA), Grandio (Voco, Cuxhaven, Germany), and Tetric EvoCeram (Ivoclar Vivadent, Schaan, Liechtenstein) are all examples of commercially available nanocomposites. ${ }^{46-48}$ 


\section{Bonding Agents}

The homogeneity and perfectly mixed adhesive properties of nanoparticles make them suitable for use as components of bonding agents. Other advantages of nanoparticles include high stress absorption, high bond strength, durable marginal seal, fluoride release, long shelf-life, and no requirement for acid etching. ${ }^{49}$ Hoshika et al., upon examining the effects of different concentrations of colloidal platinum nanoparticles, found that bond strength between resin and dentin was seen to increase when dentin was treated with a lower concentration of the material. ${ }^{50}$

\section{Impression Materials}

The addition of nanofillers in vinyl polysiloxanes was seen to improve the hyrophilic properties, flow, and accuracy of siloxane impression materials when compared with traditional vinylpolysiloxanes. ${ }^{40,41}$ Therefore, nanofillers may be integrated into conventional vinyl polysiloxanes in order to improve their properties and produce fewer voids, better model casting, and advanced accuracy. ${ }^{9,45}$ Nanotech elite H-D plus (Zhermack Italy) is an example of such an impression material that is commercially available. $^{8,20}$ This material has several advantages including high tear resistance, increased fluidity, resistance to distortion, hydrophilic properties, and snap set and heat resistance, thus reducing errors caused by micromovements. ${ }^{45,51}$

\section{NanoComposite Denture Teeth}

Although porcelain dentures exhibit high wear resistance, they are fragile, poorly connected to the denture base, and difficult to polish. Conversely, acrylic resin dentures are easy to manipulate but are highly susceptible to abrasion. Nanocomposite dentures contain uniformly dispersed nanosized filler particles and polymethylmethacrylate, which deliver superior surface structure, polishability, surface hardness, and wear resistance. $^{51}$

\section{Orthodontic Treatment}

Nanorobots can directly regulate periodontal tissues, including the gingiva, periodontal ligament, alveolar bone, and cementum, allowing fast and painless vertical repositioning, rotating, and tooth straightening in a few hours. ${ }^{29,52}$

A new stainless steel wire with an electroless nickel-phosphorous film filled with fullerene-like inorganic nanoparticles of tungsten disulfide (IF-WS $)_{2}$ nanotechnology could provide a new way to reduce friction during tooth movement. Studies evaluating the toxicity of fullerene-like nanoparticles found them to be biocompatible. Moreover, these wires were also found to combine ultra-high strength with good deformability, and exhibit resistance to corrosion and superior surface finish. ${ }^{48}$

\section{Surgical Treatment \\ Local Anesthesia}

Local anesthetic injections are commonly used in dental procedures and can cause discomfort and many associated complications, especially in pediatric patients. The use of nanotechnology in anesthesia includes delivery of an analgesic colloidal suspension containing many functional micron-sized dental nanorobot particles into the patient's gingival mucosa. Upon contact with the gingival mucosa, the mobile nanorobots migrate through the gingival sulcus, pass painlessly through the lamina propria or the layer of loose tissue on the cemento-dentinal junction, and enter into the pulp where they control nerve impulses by blocking the nerve endings. ${ }^{53}$

Upon completion of dental treatment, the dentist stimulates the nanorobots to restore all nerve impulses and leave the tooth in a similar manner. This is followed by aspiration of the tooth. Nanorobotic analgesics offer greater patient comfort, precise selectivity of analgesic effect, less side effects and complications, minimal patient anxiety and controllability and complete reversibility of the analgesic effect. ${ }^{9,20,54,55}$

\section{Nanoneedles}

Nanosized stainless steel crystals may be used in the manufacture of suture needles (commercially available examples include RK 91, Sandvik Bioline, and AB Sandvik Sweden needles), which can make oral and periodontal cell surgery possible in the near future. ${ }^{45}$

\section{Bone Replacement Materials}

Bone grafts closely reflect the chemical composition and structure of natural bone. The adhesion and growth of osteoblasts on nanophase hydroxyapatites were found to be significantly higher than those on conventional hydroxyapatite. ${ }^{56}$

Similar to other synthetics, nanophase hydroxyapatite materials reduce the risk of immune rejection and stimulate better cell growth. One such nanoproduct is made up of two bonelike components: calciumcontaining nanocrystals with the size of natural bones, and collagen to mimic the soft tissues around the natural bones (https://www.technologyreview. $\mathrm{com} / \mathrm{s} / 411048 /$ nanostructured-bone-graft/). Another nanorobotic product is made up of a mixture of nanocrystalline calcium sulfate particles and calcium sulfate hemihydrate powder, and promotes bone regeneration over a longer period of time (https://www. osseonews.com/shop/nanogen-nanotechnology-basedbone-graft). It can be used to repair bone defects caused by facial injuries and periodontal surgery. ${ }^{22}$ 


\section{Potential Future Applications \\ Dentifrobots}

Dentifrobots are nanorobots that identify and destroy pathogenic oral bacteria and prevent putrefaction, thus maintaining a healthy oral ecosystem and minimizing halitosis. They are usually made up of nanosized hydroxyapatite molecules, delivered either through mouthwash or toothpaste, and can be deactivated if ingested accidentally by the patient. ${ }^{45,57}$

Dentin hypersensitivity is relatively common and is provoked by changes in pressure that are hydrodynamically transferred to the pulp. ${ }^{55}$ The dentinal tubules of a hypersensitive tooth are twice the diameter and have eight times the surface density of non-sensitive teeth. ${ }^{58}$

The basic approach to treatment of dentin hypersensitivity involves blockage of open dentinal tubules, thus preventing external stimuli from creating fluid movements that trigger pain. Dental nanorobots can selectively identify teeth in minutes and block dentinal tubules by using natural biologic substances, thus offering patients a fast and long-lasting cure., ${ }^{3,55}$

\section{Tooth Reconstruction}

Nanodentistry plays an important role in natural tooth repair through genetic engineering, tissue engineering and regeneration, as well as production and installation a of a whole new tooth in vitro. ${ }^{29,52,59}$

The use of nanorobotic technology to manufacture and install new teeth with the same mineral and cellular components ${ }^{19}$ as the original tooth structure would greatly improve the whole treatment plan.

\section{Possible Limitations of Nanotechnology}

In principle, nanomaterials may damage the environment and negatively affect human health. However, these potential hazards may become obvious after many years as the long-term effects of nanotechnology are still unclear. ${ }^{60}$

Various factors, such as the physicochemical properties, size, and duration of exposure, govern the amount of free nanoparticles in nature. Moreover, different biological conditions, $\mathrm{pH}$, temperature, and presence of other pollutants could also interfere in interaction of nanomaterials and modify their release into the environment. ${ }^{61}$ Unfortunately, the potential effects of nanotechnologies on human health and toxicology have not been studied yet.

However, there are concerns that nanomaterials can enter the body via the lungs or skin and translocate to vital organs. Additionally, the toxicity of nanoparticles cannot be predicted from their chemical composition alone. Therefore, further research evaluating the potential risks of nanomaterials is necessary, and caution must be exercised.

\section{Nanorobotics}

Nanorobots are theoretical microscopic devices measured on the nanometer scale. They are also referred to as nanites or nanomachines, and have a diameter of $0.5-3$ microns and components that are between $1-100 \mathrm{~nm}$ in size. ${ }^{2,3,62}$

Nanorobots can respond to certain programs that allow clinicians to perform accurate procedures at the cellular and molecular levels. ${ }^{3}$ They can be operated by dentists using acoustic signals and/or electromagnetic waves. The first nanorobot was designed to crawl or dip through human tissue with navigational accuracy. Nanorobots can be used in various areas of medicine, including pharmaceutics, diagnostics, gene therapy, and dentistry. ${ }^{2}$

Nanotechnology has potential applications in various fields, as follows: environment and chemistry (filtration, catalysis), medicine (tissue engineering, drug delivery, diagnostics), energy (reduction of energy, increasing the efficiency of energy production, consumption, and recycling of batteries), communication and information, and the heavy industry. ${ }^{8}$ Nanorobots are hypothetical devices that are currently in the development stage, and can potentially be used in gerontology, dental therapy, diagnostics, pharmacology, natural immunity, atherosclerotic lesion reversal, brain injury repair, cellular damage repair, lung function improvement, and cellular DNA sequence modification in the future.

The emergence of a new field, nanotechnology, has led to growing interest in its application in the field of dentistry. ${ }^{63}$ The term was first coined in 2000 by R.A. Freitas Jr, ${ }^{9}$ who was also responsible for the introduction of tooth generation, nanomaterials, and nanorobots in dentistry. ${ }^{19}$

Nanorobots are essentially controllable machines consisting of nanoscale components ranging from 1 to $100 \mathrm{~nm}$ in size and approximately 0.5 to 3 microns in diameter. They are responsive to certain programs that enable clinicians to perform accurate procedures at the cellular and molecular levels. ${ }^{51}$ Due to their small size, nanorobots are rarely seen by the naked eye, and for their detection. ${ }^{15}$

Dental nanorobots can use specific motility mechanisms to penetrate human tissues in a manner that is efficient in terms of navigational accuracy, energy gained, and manipulation of the environment in real time. As a result, they are capable of assisting in the treatment of dental pathologies without causing harm to beneficial oral microflora. A nanocomputer that executes programmed instructions in response to local sensory stimuli can be used to control nanorobot functions. Additionally, the dentist may also provide strategic commands directly to the nanorobots via in vivo acoustic signals. ${ }^{9,55}$ 


\section{CONCLUSION}

Nanotechnology is a relatively new, rapidly developing area of science that has great potential in the effective development of new devices and nanomaterials that can be used in the management of human health. This also includes the field of dentistry, where nanotechnology is already being applied for the development of novel nanomaterials, better diagnostic and treatment plans, and tissue regeneration for the improvement and maintenance of oral health. However, despite rapid developments in this field, it is still in its initial phase and, therefore, requires further research and clinical studies.

\section{REFERENCES}

1. Gupta J. Nanotechnology applications in medicine. Tumori. 2011;94(2):206-15.

2. Kumar PS, Kumar S, Savadi RC, John J. Nanodentistry: A paradigm shift-from fiction to reality. J Indian Prosthodont Soc. 2011;11(1):1-6.

3. Dalai DR, Bhaskar DJ, R CA, Singh N, Gupta D, Bumb SS. Futuristic application of nanorobots in dentistry. Int J Adv Heal Sci. 2014;1(3):16-20.

4. Kaur J, Sharma S, Bansal A. Nanotechnologythe era of molecular dentistry. Indian J Dent Sci. 2011;3(5):80-2.

5. Feynman R. There's plenty of room at the bottom. Eng Sci. 1960;23:22-36.

6. Freitas R. Nanomedicine, volume I: basic capabilities. In:. Georgetown, TX: Landes Bioscience; 1999.

7. Praveena C, Manne P, Kalluri L, Anne R. Nanobiomaterials and their application in prosthodontics. In: Dental Applications of Nanotechnology. Cham: Springer. p. 177-201.

8. Bhardwaj A, Bhardwaj A, Misuriya A, Maroli S, Manjula S, Singh AK. Nanotechnology in dentistry: Present and future. JIOH. 2014;6(1):121-6.

9. Freitas RA. Nanodentistry. J Am Dent Assoc. 2000;131(11):1559-65.

10. Dmitrienko E, Naumova O, Fomin B, Kupryushkin M, Volkova A, Amirkhanov N, et al. Surface modification of SOI-FET sensors for label-free and specific detection of short RNA analyte. Nanomedicine. 2016;11(16):2073-82.

11. Sah AK, Vyas A, Suresh PK, Gidwani B. Application of nanocarrier-based drug delivery system in treatment of oral cancer. Artif Cells Nanomed Biotechnol. 2018;46(4):650-7.

12. Verma SK, Chauhan R. Nanorobotics in dentistry-A review. Indian J Dent 2014;5:62-70.

13. Braun A, Beisel C, Brede O, Krause F. Laser fluorescence of dentin caries covered with a novel nanofilled sealant. Lasers Med Sci. 2013;28(1):1338 .
14. Zhan C, Yang X, Yin X, Hou J. Exosomes and other extracellular vesicles in oral and salivary gland cancers. Oral Dis. 2019 (May):1-11.

15. Ummat A, Dubey A, Mavroidis C. BioNanorobotics-A Field. Inspired by nature. In: Biomimetics Biol Inspired Technol. 2006:201-26.

16. Tschoppe P, Zandim DL, Martus P, Kielbassa AM. Enamel and dentin remineralization by nanohydroxyapatite toothpastes. J Dent. 2011;39(6):430-7.

17. Han Y, Jia L, Zheng Y, Li W. Salivary exosomes: Emerging roles in systemic disease. Int J Biol Sci. 2018;14(6):633-43.

18. Parvanian S, Mostafavi SM, Aghashiri M. Multifunctional nanoparticle developments in cancer diagnosis and treatment. Sens Bio Sens Res. 2017;13:81-7.

19. Aeran H, Kumar V, Uniyal S, Tanwer P. Nanodentistry: Is just a fiction or future. J Oral Biol Craniofac Res. 2015;5(3):207-11.

20. Chauhan R, Verma SK. Nanorobotics in dentistry-A review. Indian J Dent. 2014;5(3):62-70.

21. Mouli C, Kumar M, Parthiban S. Nanotechnology in dentistry - A review. Int J Biol Med Res. 2012;3(1):1550-3.

22. Verma SK, Prabhat KC, Goyal L, Rani M, Jain A. A critical review of the implication of nanotechnology in modern practice. Natl J Maxillofac Surg.2010;1(1):41-4.

23. Lakshmi KR, Nelakurthi H, Kumar AS, Rudraraju A. Oral fluid-based biosensors: A novel method for rapid and noninvasive diagnosis. Indian J Dent Sci. 2017;9(1):60-6.

24. Najibfard K, Ramalingam K, Chedjieu I, Amaechi BT. Remineralization of early caries by Nano hydroxyapatite dentifrice. J Clin Dent. 2011;22(5):139-43.

25. Punyadeera C, Slowey PD. Saliva as an emerging biofluid for clinical diagnosis and applications of MEMS/NEMS in salivary diagnostics. In: Nanobiomaterials in Clinical Dentistry. Elsevier. p. 543-65.

26. Fung MHT, Duangthip D, Wong MCM, Lo ECM, $\mathrm{Chu} \mathrm{CH}$. Arresting dentin caries with different concentration and periodicity of silver diamine fluoride. JDR Clin Transl Res. 2016;1(2):143-52.

27. Espíndola-Castro LF, Rosenblatt A, Galembeck A, Monteiro G. Dentin staining caused by nanosilver fluoride: A comparative study. Oper Dent. 2020;710.

28. Freire PLL, Albuquerque AJR, Farias IAP, da Silva TG, Aguiar JS, Galembeck A, et al. Antimicrobial and cytotoxicity evaluation of colloidal chitosansilver nanoparticles-fluoride nanocomposites. Int J Biol Macromol. 2016;93(A):896-903.

29. Chandki R, Kala M, Kiran Kumar N, Brigit B, Banthia P, Banthia R. Nanoddentistry: Exploring 
the beauty of miniature. J Clin Exp Dent. 2012;4(2):119-24.

30. Santos VE, Vasconcelos Filho A, Targino AG, Flores MA, Galembeck A, Caldas AF, Rosenblatt A. A new "silver-Bullet" to treat caries in children - Nano silver fluoride: A randomised clinical trial. J Dent. 2014;42(8):945-51.

31. Abadi MFD, Mehrabian S, Asghari B, Namvar AE, Ezzatifar F, Lari AR. Silver nanoparticles as active ingredient used for alcohol-free mouthwash. GMS Hyg Infect Control. 2013;8(1):Doc05.

32. Roveri N, Battistella E, Bianchi CL, Foltran I, Foresti E, Iafisco M et al. Surface enamel remineralization: Biomimetic apatite nanocrystals and fluoride ions different effects. J Nanomater. 2009;2009:1-9.

33. Vano M, Derchi G, Barone A, Covani U. Effectiveness of nanohydroxyapatite toothpaste in reducing dentin hypersensitivity: A doubleblind randomized controlled trial. Quintessence Int. 2014;45(8):703-11.

34. Huang S, Gao S, Cheng L, Yu H. Combined effects of nano -hydroxyapatite and Galla chinensis on remineralization of initial enamel lesion in vitro. J Dent. 2010;38(10):811-9.

35. Huang SB, Gao SS, Yu HY. Effect of nanohydroxyapatite concentration on remineralization of initial enamel lesion in vitro. Biomed Mater. 2009;4(3):034104.

36. Kim M, Kwon H, Choi C, Kim B. Combined effects of nanohydroxyapatite and $\mathrm{NaF}$ on remineralization of early caries lesion. Key Eng Mater. 2007;330-332:1347-50.

37. Orsini G, Procaccini M, Manzoli L, Giuliodori F, Lorenzini A, Putignano A. A double-blind randomized-controlled trial comparing the desensitizing efficacy of a new dentifrice containing carbonate/hydroxyapatite nanocrystals and a sodium fluoride/potassium nitrate dentifrice. J Clin Periodontol. 2010;37(6):510-7.

38. Jena A, Kala S, Shashirekha G. Comparing the effectiveness of four desensitizing toothpastes on dentinal tubule occlusion: A scanning electron microscope analysis. J Conserv Dent. 2017;20(4):269-72.

39. Mirmohammadi H; 2018. Resin-based ceramic matrix composite materials in dentistry. In: Advances in Ceramic Matrix Composites. 2nd ed. 2nd ed Low IM, editor. Elsevier Ltd. p. 741-62.

40. Deshmukh S, Nandlal B. Evaluation of the shear bond strength of nanocomposite on carious and sound deciduous dentin. Int J Clin Pediatr Dent. 2012;5(1):25-8.

41. Van Dijken JW, Pallesen U. Four-year clinical evaluation of Class II nanohybrid resin composite restorations bonded with a one-step self-etch and a two-step etch-and-rinse adhesive. J Dent. 2011;39(1):16-25.

42. Laegreid T, Gjerdet NR, Johansson AK. Extensive composite molar restorations: 3 years clinical evaluation. Acta Odontol Scand. 2012;70(4):34452.

43. Huang S, Gao S, Cheng L, Yu H. Remineralization potential of nanohydroxyapatite on initial enamel lesions: An in vitro study. Caries Res. 2011;45(5):460-8.

44. Cheng L, Weir MD, Xu HHK, Kraigsley AM, Lin NJ, Lin-Gibson S, Zhou X. Antibacterial and physical properties of calcium-phosphate and calcium-fluoride nanocomposites with chlorhexidine. Dent Mater. 2012;28(5):573-83.

45. Jain S, Jain AP, Jain S, Gupta ON, Vaidya A. Nanotechnology: An emerging area in the field of dentistry. J Dent Sci. 2013:1-9.

46. 3M ESPE Filtek Supreme Ultra Flowable Restorative Technical Product Profile.

47. Lendenmann U, Wanner M; 2002 Ivoclar Scientific Documentation for Tetric EvoCeram.

48. Sirik ZS, Ozkol GI. Nanotechnology in dentistry. J Istanb Univ Fac Dent. 2014;48(1):79-87.

49. Priyadarsini S, Mukherjee S, Bag J, Nayak N, Mishra M; 2020. Application of nanoparticles in dentistry: Current trends. In: Nanoparticles in Medicine. Singapore: Springer. p. 55-98.

50. Hoshika S, Nagano F, Tanaka T, Wada T, Asakura K, Koshiro K, et al. Expansion of nanotechnology for dentistry: Effect of colloidal platinum nanoparticles on dentin adhesion mediated by 4-META/MMA-TBB. J Adhes Dent. 2011;13(5):411-6.

51. Kanaparthy R, Kanaparthy A. The changing face of dentistry: Nanotechnology. Int J Nanomed. 2011;6:2799-804.

52. Ingle E, Gopal K. Nanodentistry. A hype or hope. J Oral Heal Comm Dent. 2011;5(2):64-7.

53. Florez FLE, Pathak Y, Preuss C; 2012. Orally delivered nanoparticle drug delivery systems for dental applications and their toxicity on systemic organs. In: Nanobiomaterials in Clinical Dentistry. 2nd ed Subramani K, Ahmed W, editors. Elsevier Inc. p. 595-616.

54. Rao A, Tummala M, Elkanti S, Mikkilineni M. Nanodentistry: New buzz in dentistry. Eur J Gen Dent. 2013;2(2):109.

55. Shetty NJ, Swati P, David K. Nanorobots: Future in dentistry. Saudi Dent J. 2013;25(2):49-52.

56. Bhullar-Sukhwinder K, Gazioglu-Ruzgar D, SaberSamandari S, Sadighi M, Ahadian S, Ramalingam M. Impact of nanophase hydroxyapatite-based biomaterials on tissue engineering. J Bionanosci. 2018;12(4):469-477(9). 
57. Babel S, Mathur S. Nanorobotics-headway towards dentistry. JRST. 2011;1(3).

58. Şuhani MF, Băciuţ G, Băciuţ M, Şuhani R, Bran S. Current perspectives regarding the application and incorporation of silver nanoparticles into dental biomaterials. Clujul Med. 2018;91(3):274-9.

59. Ozak ST, Ozkan P. Nanotechnology and dentistry. Eur J Dent. 2013;7(1):145-51.

60. Larsson S, Jansson M, Boholm Å. Expert stakeholders' perception of nanotechnology: Risk, benefit, knowledge, and regulation. J Nanopart Res. 2019;21(3):57.

61. Gambhir R, Sogi G, Nirola A, Sekhon T, Kakar H, Brar R. Nanotechnology in dentistry: Current achievements and prospects. J Orofac Sci. 2013;5(1):9.

62. S, Kirti BP, Khuller N, Ma BA. Nanorobotics - Beyond the microscope in periodontics. J Stomatognath Sci. 2015;5(1):24-7.

63. Abhilash M. Nanorobots. Int J Pharm Biol Sci. 2010;1(1):1-10.

(Accepted June 19, 2020) 